\title{
ChemComm
}

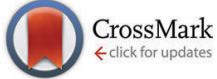

Cite this: Chem. Commun., 2016, 52,7486

Received 22nd March 2016 , Accepted 13th May 2016

DOI: $10.1039 / \mathrm{c} 6 \mathrm{cc0} 02489 \mathrm{k}$

www.rsc.org/chemcomm

\section{A non-topological mechanism for negative linear compressibility $\dagger$}

\author{
Jack Binns, ${ }^{\text {ab }}$ Konstantin V. Kamenev, ${ }^{c}$ Katie E. R. Marriott, ${ }^{d}$ Garry J. McIntyre, ${ }^{b}$ \\ Stephen A. Moggach, ${ }^{a}$ Mark Murrie ${ }^{\star d}$ and Simon Parsons ${ }^{* a}$
}

\begin{abstract}
Negative linear compressibility (NLC), the increase in a unit cell length with pressure, is a rare phenomenon in which hydrostatic compression of a structure promotes expansion along one dimension. It is usually a consequence of crystal structure topology. We show that the source of NLC in the Co(II) citrate metal-organic framework UTSA16 lies not in framework topology, but in the relative torsional flexibility of Co(॥)-centred tetrahedra compared to more rigid octahedra.
\end{abstract}

The second law of thermodynamics requires that when pressure is applied to a crystal its unit cell volume always decreases. ${ }^{1}$ In virtually all crystalline materials this volume reduction is achieved through a shortening in all three unit cell lengths. But since the individual cell dimensions are not thermodynamic variables, exceptions to this rule are possible, and in some rare and anomalous materials one or two cell dimensions increase under pressure. This behaviour is called negative linear compressibility (NLC), ${ }^{2}$ and potential applications include high-performance pressure sensors or body armour, ${ }^{3}$ composite engineering materials, ${ }^{4}$ force amplifiers $^{5}$ and optical devices for deep-sea environments. ${ }^{6}$

The source of NLC is "supramolecular", reflecting the way in which basic structural building blocks, such as metal-based tetrahedra and octahedra or larger secondary bonding units, are linked together. The structural features that give rise to NLC behaviour and the range of materials for which the phenomenon is observed have been recently reviewed. ${ }^{2}$ The most common structural feature associated with NLC is the 'wine-rack'

\footnotetext{
${ }^{a}$ EaSTCHEM School of Chemistry and Centre for Science at Extreme Conditions, The University of Edinburgh, Joseph Black Building, The King's Buildings, West Mains Road, Edinburgh EH9 3FJ, UK. E-mail: S.Parsons@ed.ac.uk

${ }^{b}$ Australian Nuclear Science and Technology Organisation, New Illawarra Road, Lucas Heights, NSW 2234, Australia

${ }^{c}$ School of Engineering and Centre for Science at Extreme Conditions, The University of Edinburgh, Erskine Williamson Building, The King's Buildings, Peter Guthrie Tait Road, Edinburgh, EH9 3JJ, UK

${ }^{d}$ WestCHEM, School of Chemistry, University of Glasgow, Glasgow, G12 8QQ, UK. E-mail: Mark.Murrie@glasgow.ac.uk

$\dagger$ Electronic supplementary information (ESI) available. CCDC 1447804-1447807. For ESI and crystallographic data in CIF or other electronic format see DOI: $10.1039 / \mathrm{c} 6 \mathrm{cc} 02489 \mathrm{k}$
}

motif ${ }^{6,7}$ found in a number of materials such as $\mathrm{Ag}_{3}\left[\mathrm{Co}(\mathrm{NC})_{6}\right]$, $\mathrm{KMn}\left[\mathrm{Ag}(\mathrm{CN})_{2}\right]_{3}$, and methanol monohydrate, as well as a number of metal-organic frameworks (MOFs). ${ }^{8-13}$ Compression causes one diagonal non-bonded distance of the wine-rack to increase while the other decreases. ${ }^{14}$

Other motifs have also been found to lead to NLC. ${ }^{14,15}$ The largest NLC effect has been reported for the cyanide framework zinc dicyanoaurate, $\mathrm{Zn}\left[\mathrm{Au}(\mathrm{CN})_{2}\right]_{2}$ which contains a hexagonal honeycomb pore. The pores are linked by helical $\mathrm{Au} \cdots \mathrm{Au}$ 'springs' which compress along the direction of the spring, causing the coupled framework to expand along one of the perpendicular directions. The $3.3 \%$ increase in the length of the corresponding axis up to $1.6 \mathrm{GPa}(\sim 15800 \mathrm{~atm})$ characterises an axial compressibility, $\beta_{\mathrm{NLC}}$, of $-42(5) \mathrm{TPa}^{-1} \cdot{ }^{15}$

We now report NLC behaviour in the MOF UTSA-16, ${ }^{16,17}$ $\left(\left[\mathrm{KCO}_{3}(\mathrm{cit})(\mathrm{Hcit})\left(\mathrm{H}_{2} \mathrm{O}\right)_{2}\right] \cdot 8 \mathrm{H}_{2} \mathrm{O}\right)_{n},\left(\mathrm{cit}=\mathrm{C}_{6} \mathrm{H}_{4} \mathrm{O}_{7}\right.$; UTSA $=$ University of Texas at San Antonio). We show that NLC arises in this compound not through changes in topological metrics, ${ }^{14}$ but through flexing of the metal-based polyhedra themselves.

Crystals of UTSA-16 were prepared by solvothermal reaction in a Teflon-lined Parr acid digestion bomb according to ref. 17. Single crystal diffraction data were collected at room temperature at ambient pressure and at 0.3, 0.5 and 1.0 GPa. Details of procedures and data analysis are given in the ESI $\dagger$ (Section S1) ${ }^{18-26}$ Crystallographic data can be obtained from the Cambridge Crystallographic Data Centre, quoting entries CCDC 1447804-1447807.

UTSA-16 consists of two crystallographically independent $\mathrm{Co}^{2+}$ centres and one unique citrate ligand. Co1 and the alkoxy oxygen atom (O3) from the citrate form $S_{4}$-symmetric $\mathrm{Co}_{4} \mathrm{O}_{4}$ cubanes. Carboxylate oxygen atoms $\mathrm{O} 1, \mathrm{O} 4$ and $\mathrm{O} 6$ from each citrate bind to the three Co atoms connected to O3, making each Co1 centre six coordinate (Fig. 1(a) and (b)).

A second, tetrahedral, building block is formed by Co2 which is located on a two-fold axis and coordinated to one pair of equivalent carboxylate atoms (O2) from the same cubane, and another pair $(\mathrm{O} 7)$ derived from different cubanes. Potassium cations (K1) also reside on two-fold axes and bind to the same 
a)

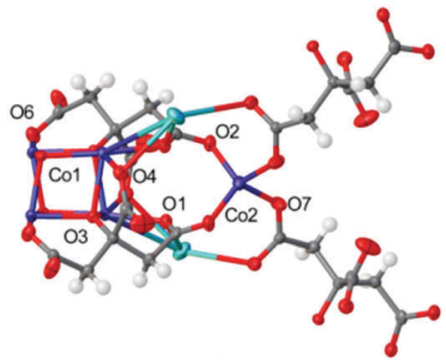

c)

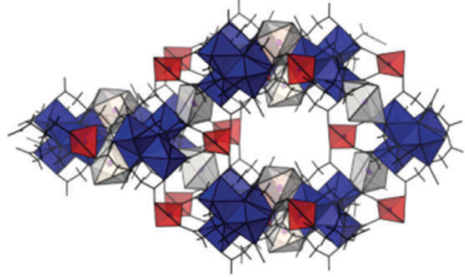

b)

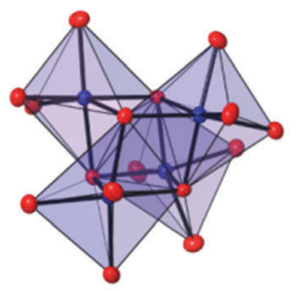

d)

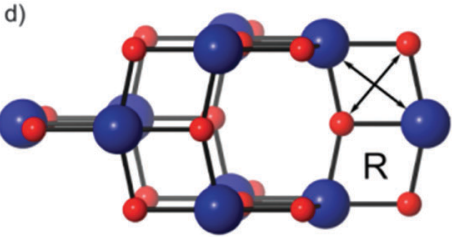

Fig. 1 (a) Citrate-encapsulated cubane units are linked by Co tetrahedra; (b) $\mathrm{Co1}$ atoms are linked by oxygen atoms O1, O3, O4, and O6 to form $\mathrm{S}_{4}$-symmetric $\mathrm{CO}_{4} \mathrm{O}_{4}$ cubanes; (c) polyhedral representation of UTSA-16, $\mathrm{CO}_{4} \mathrm{O}_{4}$ units are shown in blue, Co tetrahedra in red, and irregular $\mathrm{K}$ polyhedra in white; (d) structure of anatase, 141/amd, (Ti - Blue, O - Red) whose topology is analogous to UTSA-16. 'Wine-rack' rings indicated with ' $\mathrm{R}$ ', diagonals indicated with arrows.

carboxylate oxygen atoms (O1, O4, and O6) coordinated to Co1. The eight-fold coordination sphere of $\mathrm{K} 1$ is completed by two equivalent disordered water molecule sites (O80/O81). Each cubane thus binds to six tetrahedra based on Co2 (two through pairs of $\mathrm{O} 2$ and four through $\mathrm{O} 7$ ), while each tetrahedron binds to three cubanes.

Overall the topology of the structure is analogous to anatase, a form of $\mathrm{TiO}_{2}$, where the cubanes correspond to titanium and the tetrahedra correspond to oxides (Fig. 1(c) and (d)). The

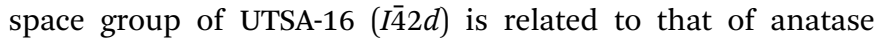
$\left(I 4_{1} /\right.$ amd $)$ by the loss of inversion symmetry as the result of the replacement of spherically symmetric atoms with lower symmetry cubanes and tetrahedra. Successive rhomboid rings, indicated by $\mathrm{R}$ in Fig. 1(d) and formed by the cubanes and tetrahedra, generate a wine-rack motif.

Single-crystal diffraction data were collected at ambient pressure and at 0.3(1), 0.5(1), 1.0(1) and 1.7(1) GPa. Fluorinert FC70 was used as a hydrostatic medium at high pressure because it consists of large perfluorohydrocarbons which do not penetrate the framework at pressure, thus avoiding complications of host-guest interaction $\mathrm{s}^{27-29}$ and enabling the native mechanical properties of the framework to be characterized.

Up to $1.0 \mathrm{GPa}$, the unit cell volume decreases by $332.0(6) \AA^{3}$ (6.4\%). Fitting the pressure-volume data to a second-order Birch-Murnaghan equation of state ${ }^{30}$ yields a bulk modulus of 13.4(12) $\mathrm{GPa}\left(\beta_{\mathrm{V}}=75(9) \mathrm{TPa}^{-1}\right)$, which is comparable to other framework materials. ${ }^{15,31}$ FC70 becomes non-hydrostatic beyond $1 \mathrm{GPa}$, and above this pressure the material lost crystallinity. Normalised changes in unit cell dimensions and volumes are given in Fig. 2. The reduction in volume is due to compression along the $a$ and $b$-axes, which shorten by $0.4580(6) \AA(-3.5 \%$, $\left.\beta_{a}=34.04 \mathrm{TPa}^{-1}\right)$. The $c$-axis is subject to NLC, increasing in length by $0.1429(15) \AA$, a change of $+0.5 \%\left(\beta_{c}=-4.74 \mathrm{TPa}^{-1}\right)$.

The interest in this pattern of compressibility lies not so much in its magnitude as in its mechanism. If UTSA-16 were a

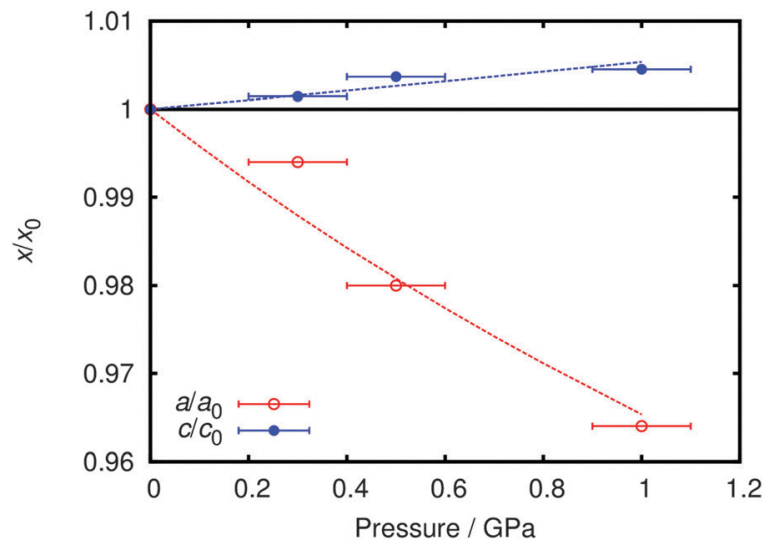

Fig. 2 Normalised changes in unit cell dimensions with pressure. 2nd-order Birch-Murnaghan equations of state are represented as dashed lines. The error bars in the vertical axis are smaller than circles used for the data points.

typical NLC material either the diagonal cubane-cubane or tetrahedron-tetrahedron distances (indicated by arrows in Fig. 1(d)) within the wine-rack motif would be expected to increase. Actually they both decrease, by $0.121(2)$ and $0.261(2) \AA$, respectively. Neither does anatase itself exhibit NLC. ${ }^{32}$ The unusual piezomechanical behaviour of UTSA-16 must therefore arise from changes within the cubanes or tetrahedra themselves, rather than as a result of topology.

On increasing pressure, no statistically significant changes in either bond distances or angles were observed within the cubane units or the citrate ligands. Tethering the linking carboxylate group to the cubane unit also prevents any significant torsion in these groups over the pressure range.

By contrast, substantial changes can be seen in tetrahedra based on $\mathrm{Co} 2$ (Fig. 3). The volume of the tetrahedra decreases by $0.185 \AA^{3}(-4.8 \%)$ up to $1.0 \mathrm{GPa}$. Though there is no change 
a)

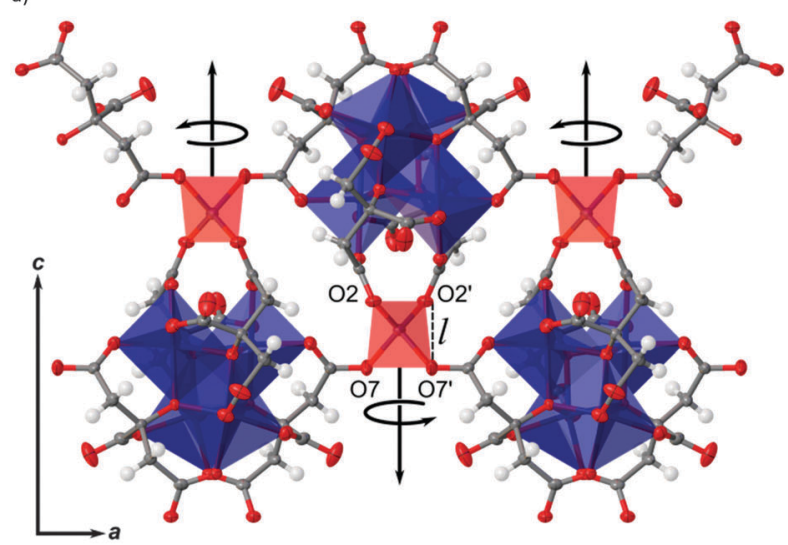

b)

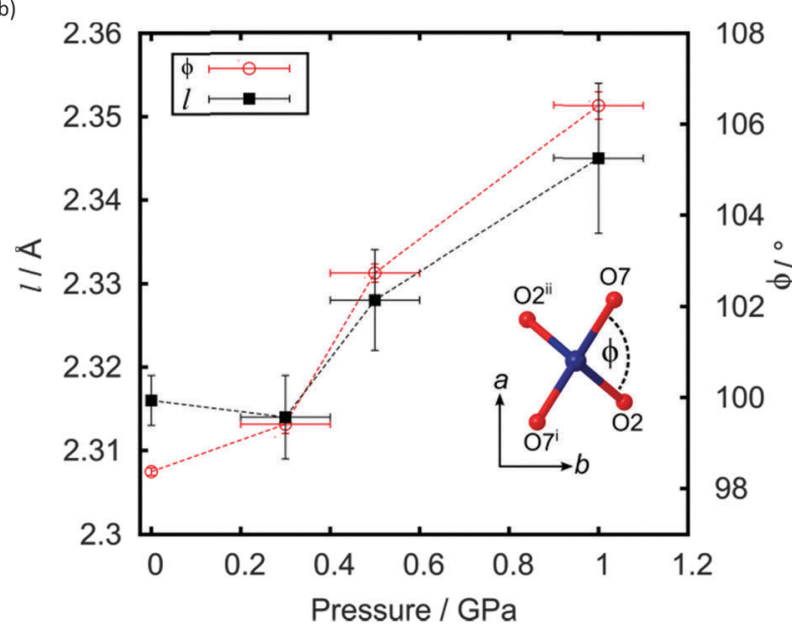

Fig. 3 (a) NLC arises along the $c$ axis by the elongation of the Co tetrahedra, a result of the twisting of the $\mathrm{O}-\mathrm{Co}-\mathrm{O}$ planes. In order to maintain $\mathrm{Co}-\mathrm{O}$ bond lengths, the tetrahedron height, l, must increase: (b) increase in dihedral angle $\phi$ and consequential increase in tetrahedron height / with increasing pressure.

in the $\mathrm{Co} 2-\mathrm{O}$ bond distances, there is a large twisting motion between the planes $\mathrm{O} 2-\mathrm{Co}-\mathrm{O} 2{ }^{\prime}$ and $\mathrm{O} 7-\mathrm{Co}-\mathrm{O}^{\prime}$ 'increasing the dihedral angle $\phi$ from $98.37(8)^{\circ}$ to $106.4(3)^{\circ}$ (see ESI, $\dagger$ Section S2). The twisting motion reduces the distance between linked cubanes along one axis, and this contraction is replicated in an orthogonal direction by symmetry-related tetrahedra. This concerted motion is the mechanism for compression in the $a$ and $b$ directions (Fig. 3(a)).

Changes in the height of the tetrahedron projected on the $c$ axis $(l)$ as $\phi$ varies with pressure are shown in Fig. 3(b). It can be seen that the torsional flexing leads to elongation of the tetrahedra along $c$, and this, rather than expansion in one of the wine-rack dimensions, is the source of the NLC effect.

The quantitative relationship of the geometric changes at $\mathrm{Co} 2$ with the $c$-axis length can be analysed (ESI, $\uparrow$ Section S3) with reference to Fig. 4, which shows a simplified structure consisting of the $\mathrm{Co} 2$ tetrahedra, the centroids of the $\left[\mathrm{Co}_{4} \mathrm{O}_{4}\right]$ cubanes (X1) and the potassium ions (K1). The structure along the $c$-axis can be divided into sets of three zones labelled A, B and $\mathrm{C}$ in Fig. 4. The length of zone B corresponds to the height

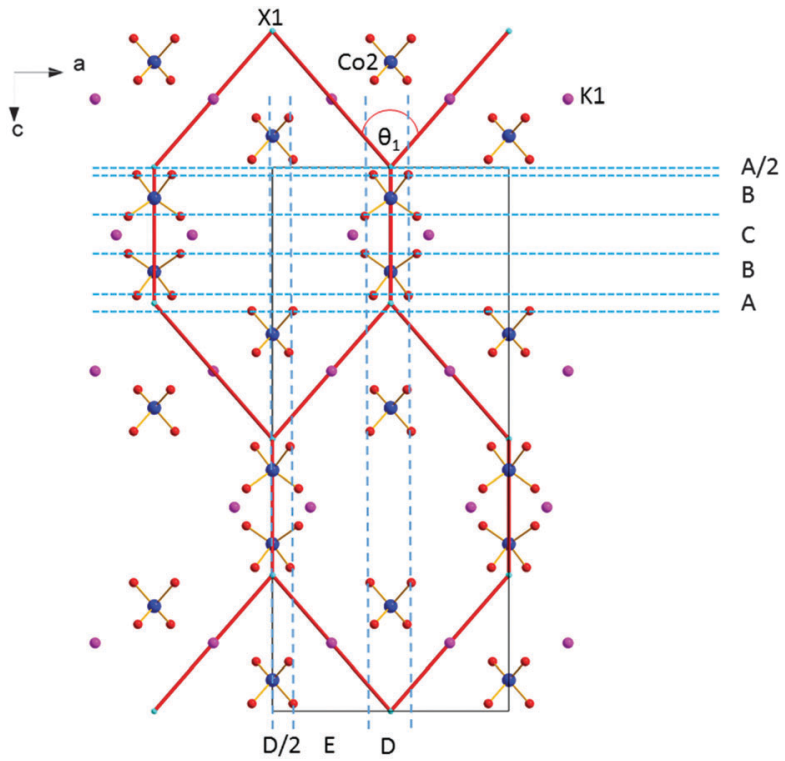

Fig. 4 Simplified view of the structure showing only the tetrahedra based on $\mathrm{Co} 2$, the centroids of the Co1-based cubanes (X1) and the potassium ions (K1). Division of the $C$-axis into regions $A, B$ and $C$, and division of the axis into regions $\mathrm{D}$ and $\mathrm{E}$ is also shown. The diamondoid lattice referred to in the text is shown in red.

of the Co2 tetrahedron projected onto $c$ (the $l$ of Fig. 3); at ambient pressure this is $2.3164 \AA$, increasing to $2.3452 \AA$ at $1 \mathrm{GPa}$, a difference of $+0.0288 \AA$. The total contribution of all the $\mathrm{CoO}_{4}{ }^{2-}$ anions in the unit cell to the NLC effect seen along $c$ is $8 \times 0.0288=0.2304 \AA$, which is larger than the change in $c$-axis length actually observed (0.1429 $\mathrm{A}$ ) (see ESI, $\dagger$ Section S3). The difference is ascribable to distortion of the coordination spheres around the potassium ions leading to the overall compression $(-0.0219 \AA)$ occurring in zones A and C. Though the change in the $\mathrm{A}+\mathrm{C}$ zones is similar in magnitude to that seen in $\mathrm{B}$, there are only four of them whereas there are eight $\mathrm{B}$ zones. The NLC effect is thus the result of expansion of the more numerous Co2 tetrahedra outweighing the compression at K1. A similar analysis along the $a$-direction (ESI, $\dagger$ Section S4), using zones D and E in Fig. 4 shows that compression of the Co2 tetrahedra in the $a$-direction $(-0.1719 \AA=D)$ accounts for around $75 \%$ of the shortening observed in this axis.

The compression of UTSA-16 can also be analysed from a topological viewpoint using a diamondoid lattice defined by nodes positioned at the centroids of the $\left[\mathrm{Co}_{4} \mathrm{O}_{3}\right]$ cubanes and outlined in red in Fig. 4 (ESI, $\dagger$ Section S5). Compression of the framework struts results in a positive linear compression (PLC, $\left.\beta_{\text {strut }}=+12.1 \mathrm{TPa}^{-1}\right)$, while reduction of the angle $\theta_{1}$ gives rise to NLC along $c$ and PLC along $a$. The contributions of the angular change along $a$ and $c$ are $\beta_{a \theta}=+23.19 \mathrm{TPa}^{-1}$ and $\beta_{c \theta}=$ $-17.08 \mathrm{TPa}^{-1}$, respectively, NLC occurring because the axis elongation brought about by changes in angle outweighs the compression effect. Nevertheless, consistent with the torsional flexing mechanism proposed above, the changes occurring in the internal geometry of the $\mathrm{Co} 2$ tetrahedron contribute approximately $85 \%$ to both $\beta_{a \theta}$ and $\beta_{c \theta}$. 
The upper limit of the NLC effect was determined in this study by the pressure limit of the hydrostatic medium used. The twisting which occurs at $\mathrm{Co} 2$ represents a step away from tetrahedral towards square planar coordination. However, while $<\mathrm{O} 7-\mathrm{Co} 2-\mathrm{O}^{\mathrm{i}}$ decreases by almost $3^{\circ}$ in response to pressure, the opposing $<\mathrm{O} 2-\mathrm{Co} 2-\mathrm{O} 2^{\mathrm{ii}}$ angle is fixed $\left(118.59(17)^{\circ}\right.$ at $0 \mathrm{GPa}$ and $118.6(5)^{\circ}$ at $1 \mathrm{GPa}$ ) by binding to a single rigid cubane. The framework connectivity is thus expected to place a physical limit on the maximum distortion attainable.

The reduction in the tetrahedral volume occurs through changes in the 'softer' torsion angles rather than by compression of the more rigid $\mathrm{Co}-\mathrm{O}$ bonds or $\mathrm{Co}-\mathrm{O}-\mathrm{Co}$ angles. The relative flexibility of the Co2 tetrahedra versus the Co1-based octahedra arises from both the effect of ligand-sharing within the encapsulated cubanes as well as the inherently greater flexibility in tetrahedral coordination spheres as a result of lower steric hindrance and a smaller crystal field splitting. This flexibility is reflected in the far wider distribution of $\mathrm{O} / \mathrm{N}-\mathrm{Co}-\mathrm{O} / \mathrm{N}$ angles adopted within tetrahedral complexes compared to octahedral complexes for structures reported in the Cambridge Structural Database (ESI, $\dagger$ Section S6). ${ }^{33}$

In conclusion, the framework material UTSA-16 has been shown to exhibit negative linear compressibility along the tetragonal $c$ axis. This behaviour is not a result of the network connectivity, as shown in other MOFs, but due to distortions within torsionally flexible cobalt(II) tetrahedra which link together rigid $\mathrm{Co}_{4} \mathrm{O}_{4}$ units based on distorted cobalt(II) octahedra. The soft torsional distortion allows the framework to compress in the $a$ and $b$ directions while expanding along $c$.

The mechanism of NLC in UTSA-16 illustrates how the modular nature of MOF structures might be exploited in the design of new materials with novel responses to high pressure. Framework materials are a fruitful subject for high-pressure research in part because all components within the structure are linked together by strong bonds, and there is no option for the effects of pressure simply to be 'absorbed' by weak and deformable intermolecular interactions as they are in molecular solids. This feature leads to much more substantial effects on primary bonding parameters than is seen in molecular coordination complexes. For example, in cristobalite analogues $\mathrm{BEO}_{4}(\mathrm{E}=\mathrm{P}, \mathrm{As})$ linear compressibilities are determined by tilting of rigid tetrahedra because $\mathrm{B}-\mathrm{O}-\mathrm{E}$ bending carries a lower energy than compression of $\mathrm{B}-\mathrm{O}$ or $\mathrm{E}-\mathrm{O}$ bonds. ${ }^{2,34}$ Similarly, extreme compressibility in $\mathrm{LnFe}(\mathrm{CN})_{6},{ }^{35}$ occurs because structural components acting as gears and torsion springs are coupled by strong framework bonds.

UTSA-16 is a framework material where a rigid framework component is combined with one which is much more deformable. The deformable component plays the same role at high pressure as intermolecular interactions do in molecular complexes, and essentially all the effects of pressure are focussed onto one small component of the framework, which then undergoes substantial deformation. In the case of the material studied here a torsional deformation in a tetrahedral centre leads to compression in two directions but expansion in the third, and because this deformation component is connected into a framework, this distortion is propagated throughout the crystal structure, leading to the overall NLC effect. This non-topological mechanism for NLC opens the way to new design strategies for NLC materials, but also forms a strategy for exploring other novel responses to compression.

We thank The University of Edinburgh, EPSRC (DTA, EP/J018147/1 and EP/K033646/1), and the Australian Government for funding. We also thank the referees for their helpful comments.

\section{Notes and references}

1 R. Gatt and J. N. Grima, Phys. Status Solidi RRL, 2008, 2, 236-238.

2 A. B. Cairns and A. L. Goodwin, Phys. Chem. Chem. Phys., 2015, 20449-20465.

3 F.-X. Coudert, Chem. Mater., 2015, 27, 1905-1916.

4 J. S. O. Evans, J. Chem. Soc., Dalton Trans., 1999, 3317-3326.

5 Z. G. Nicolaou and A. E. Motter, Nat. Mater., 2012, 11, 608-613.

6 R. H. Baughman, S. Stafström, C. Cui and S. O. Dantas, Science, 1998, 279, 1522-1524.

7 R. H. Baughman and D. S. Galvão, Nature, 1993, 365, 735-737.

8 A. L. Goodwin, D. A. Keen and M. G. Tucker, Proc. Natl. Acad. Sci. U. S. A., 2008, 105, 18708-18713.

9 A. B. Cairns, A. L. Thompson, M. G. Tucker, J. Haines and A. L. Goodwin, J. Am. Chem. Soc., 2011, 134, 4454-4456.

10 A. D. Fortes, E. Suard and K. S. Knight, Science, 2011, 331, 742-746.

11 W. Li, M. R. Probert, M. Kosa, T. D. Bennett, A. Thirumurugan, R. P. Burwood, M. Parinello, J. A. Howard and A. K. Cheetham, J. Am. Chem. Soc., 2012, 134, 11940-11943.

12 J. M. Ogborn, I. E. Collings, S. A. Moggach, A. L. Thompson and A. L. Goodwin, Chem. Sci., 2012, 3, 3011-3017.

13 W. Cai and A. Katrusiak, Nat. Commun., 2014, 5, 4337.

14 J. N. Grima, D. Attard, R. Caruana-Gauci and R. Gatt, Scr. Mater., 2011, 65, 565-568.

15 A. B. Cairns, J. Catafesta, C. Levelut, J. Rouquette, A. van der Lee, L. Peters, A. L. Thompson, V. Dmitriev, J. Haines and A. L. Goodwin, Nat. Mater., 2013, 12, 212-216.

16 S. Xiang, Y. He, Z. Zhang, H. Wu, W. Zhou, R. Krishna and B. Chen, Nat. Commun., 2012, 3, 954.

17 S. C. Xiang, X. T. Wu, J. J. Zhang, R. B. Fu, S. M. Hu and X. D. Zhang, J. Am. Chem. Soc., 2005, 127, 16352-16353.

18 APEX Suite, Bruker-AXS, Madison, Wisconsin, 2007.

19 G. M. Sheldrick, $S A D A B S$, University of Göttingen, Germany, 2008.

20 A. Dawson, D. R. Allan, S. Parsons and M. Ruf, J. Appl. Crystallogr., 2004, 37, 410-416.

21 S. Parsons, SHADE, University of Edinburgh, UK, 2004.

22 A. Altomare, G. Cascarano, C. Giacovazzo and A. Guagliardi, J. Appl. Crystallogr., 1993, 26, 343-350.

23 P. W. Betteridge, J. R. Carruthers, R. I. Cooper, K. Prout and D. J. Watkin, J. Appl. Crystallogr., 2003, 36, 1487.

24 L. Merrill and W. A. Bassett, Rev. Sci. Instrum., 1974, 45, 290-294.

25 G. J. Piermarini, S. Block, J. Barnett and R. Forman, J. Appl. Phys., 1975, 46, 2774-2780.

26 R. J. Angel, M. Alvaro and J. Gonzalez-Platas, Z. Kristallogr. - Cryst. Mater., 2014, 229, 405-419.

27 D. Fairen-Jimenez, S. Moggach, M. Wharmby, P. Wright, S. Parsons and T. Duren, J. Am. Chem. Soc., 2011, 133, 8900-8902.

28 A. J. Graham, D. R. Allan, A. Muszkiewicz, C. A. Morrison and S. A. Moggach, Angew. Chem., 2011, 123, 11334-11337.

29 S. A. Moggach, T. D. Bennett and A. K. Cheetham, Angew. Chem., Int. Ed., 2009, 48, 7087-7089.

30 F. Birch, J. Geophys. Res.: Solid Earth, 1986, 91, 4949-4954.

31 K. J. Gagnon, C. M. Beavers and A. Clearfield, J. Am. Chem. Soc., $2013,135,1252-1255$.

32 T. Arlt, M. Bermejo, M. Blanco, L. Gerward, J. Jiang, J. S. Olsen and J. Recio, Phys. Rev. B: Condens. Matter Mater. Phys., 2000, 61, 14414-14419.

33 F. H. Allen, Acta Crystallogr., Sect. B: Struct. Sci., 2002, 58, 380-388. 34 J. Haines, C. Chateau, J. M. Léger, C. Bogicevic, S. Hull, D. D. Klug and J. S. Tse, Phys. Rev. Lett., 2003, 91, 015503.

35 S. G. Duyker, V. K. Peterson, G. J. Kearley, A. J. Studer and C. J. Kepert, Nat. Chem., 2016, 8, 270-275. 